\title{
Management Fads, Pedagogies, and other Soft Technologies
}

\author{
Jonathan Bendor \\ Graduate School of Business, Stanford University, Stanford, CA 94305 \\ Bernardo A. Huberman and Fang Wu \\ HP Labs, Palo Alto, CA 94304
}

November 21, 2018

\begin{abstract}
We present a model for the diffusion of management fads and other technologies which lack clear objective evidence about their merits. The choices made by non-Bayesian adopters reflect both their own evaluations and the social influence of their peers. We show, both analytically and computationally, that the dynamics lead to outcomes that appear to be deterministic in spite of being governed by a stochastic process. In other words, when the objective evidence about a technology is weak, the evolution of this process quickly settles down to a fraction of adopters that is not predetermined. When the objective evidence is strong, the proportion of adopters is determined by the quality of the evidence and the adopters' competence.
\end{abstract}




\section{Introduction}

In domains such as management and education, organizational practices often seem to come and go in puzzling ways. They are introduced with fanfare, but then they diffuse with little evidence that they work well. While they are sometimes discarded at later times, they are so with little conclusive evidence about their performance. Consider, for example, Quality Circles. Possibly as many as 90 percent of the Fortune 500 companies had adopted QCs by 1985 , but by 1987 "more than $80 \%$ of the Fortune 500 companies that adopted QCs in the 1980s had abandoned them by 1987" Abr96 pp. 256]. Yet one is hard pressed to find hard evidence of their impact, even after the fact.

Fads are so common in American education that one observer reports that "School leaders in a whimsical mood sometimes play a parlor game called 'Spot That Jargon,' in which the goal is to name as many past educational fads as possible. The list is usually impressive." Las98, MC04

This paper examines the diffusion of such innovations or ideas. We call them soft technologies, not because of their physical properties but because evidence for or against them is equivocal, inconclusive, or even nonexistent. We contend that the choices made by adopters are quasi-rational: they reflect both an attempt to assess the imperfect data surrounding such innovations as well as a reliance on social cues, i.e., what peers have done. We argue that these two elements are linked by what could be called Festinger's Hypothesis: the more equivocal the evidence, the more people rely on social cues [Fes54 pp. 118].

In this paper we present a model that considers soft technologies as those for which (1) the objective evidence is weak and (2) people rely heavily on the prior choices of people in similar roles. We then show that the dynamics of the model leads to outcomes that appear to be deterministic in spite of being governed by a stochastic process. In other words, when the objective evidence for the adoption of a soft-technology is weak, any sample path of this process quickly settles down to a fraction of adopters that is not predetermined by the initial conditions: ex ante, every outcome is just as (un)likely as every other. In the case when the objective evidence is strong, the process settles down to a value that is determined by the quality of the evidence. In both cases the proportion of adopters of the technology never settles into either zero or one.

\section{$1.1 \quad$ Related Work}

In the most highly developed mathematical models of fads - economic theories of "herding" - decision makers also use social cues but do so in perfectly rational ways, via Bayesian updating. ${ }^{1}$ Though we agree that social cues matter we think that the premise of Bayesianism exaggerates the rationality of agents facing the difficult decision of whether or not to adopt a soft technology. In particular,

\footnotetext{
${ }^{1}$ There is now a large literature on informational cascades triggered by fully rational agents: see the annotated bibliography of Bikhchandani, Hirshleifer and Welch, available on the Web [BHW96]. For seminal papers in this line of work see Ban92 BHW92 Wel92].
} 
there is little evidence for the claim that people are perfect Bayesians. A leading experimental economist summarizes the evidence as follows:

Much research in cognitive psychology suggests that the way in which people form judgments of probability departs systematically from the laws of statistics and from Bayesian updating. (This should not be surprising, because there is no reason to think that evolution of brain processes like memory, language, perception, categorization, and reasoning would have adapted us to use a rule that Bayes only "discovered" a couple of hundred years ago.) Some research points toward systematic departures, or "biases", which spring from a small number of "heuristics", like anchoring, availability, and representativeness Cam98, pp. 171].

Thus, the theoretical value of herding models - the intriguing demonstration that what appears to be conformity behavior in the aggregate is consistent with perfectly rational action of individuals - should not be confused with empirical confirmation of its micro-postulates. As a purely theoretical point it is interesting to recognize that perfect information-processing by individual agents is, under certain circumstances, consistent with conformity-like behavior. But we suspect that to the extent that such models receive empirical support, the support will be "weak" in the sense that the data on conformity or herding will also be consistent with a wide variety of other sensible-though-suboptimal forms of individual information-processing. Again, Camerer's assessment of Bayesianism is pertinent:

As a descriptive theory, Bayesian updating is weakly grounded in the sense that there is little direct evidence for Bayesian updating which is not also consistent with much simpler theories. Most of the evidence in favor of Bayesian updating boils down to the fact that if new information favors hypothesis $\mathrm{A}$ over $\mathrm{B}$, then the judged probability of A, relative to $\mathrm{B}$, rises when the information is incorporated. This kind of monotonicity is consistent with Bayesian updating but also with a very wide class of non-Bayesian rules (such as anchoring on a prior and adjusting probabilities up or down in light of the information) [Cam98 pp. 171].

In this paper we propose a model that is consistent with all of Camerer's observations and so is an alternative to canonical herding models. Thus our agents exhibit normatively desirable and empirically plausible monotonicity properties: in particular, the more the social cues favor innovation A over B, the more likely it is that an agent will select A, ceteris paribus. Yet the reasoning that underlies such choices is adaptively rational rather than fully rational. Moreover, unlike many adaptive models of fads, the present model generates analytical solutions, not just computational ones. ${ }^{2}$

\footnotetext{
${ }^{2}$ Many-perhaps most-adaptive models of fads are what has come to be called "agentbased models" and it is virtually a defining feature of such models that they be computational. (For a survey of agent-based models, including several applied to fads, see MW02.)
} 


\section{The Model}

A world in which people can make mistakes (e.g., adopt an inferior method of instruction, partly because many other school districts have already done so) and where they are influenced by the possibly erroneous, possibly correct choices of similar decision makers is inherently probabilistic. Hence our model is built around a probabilistic choice process.

We assume that two alternatives, $\mathrm{A}$ and $\mathrm{B}$, diffuse through a population of decision makers. ${ }^{3}$ In every period one decision maker makes up his mind about whether to adopt A or B; this choice is final. (In this sense the formulation is like most "contagion" models.) The diffusion continues until everyone in the population has selected either A or B. To get the process going, we assume that initially $(t=0)$ at least one person has made a choice, i.e., at least one person already champions either A or B. However, we allow for the possibility that either option may have multiple initial champions. (One could regard these early champions as the inventors of the two alternatives.) A useful benchmark case to keep in mind is a fair start, in which A and B are backed by the same number of initial champions. The numbers of A- and B-champions at $t=0$ will be denoted by $m_{0}$ and $n_{0}$.

The heart of the model is how agents decide on which option to adopt. As noted earlier, we assume that there are two components to the adoption decision. The first is based on individual judgment; the second, on social influence. Regarding the first component, we assume that a person isolated from social influence would choose the objectively superior option (labeled A in our model) with probability $p$. Thus $p$ reflects the quality of the evidence about the relative merits of A versus B, plus whatever a priori bias (possibly due to a folk theory) exists. If A's superiority is obvious then $p$ will be close to 1 ; if the two alternatives are nearly interchangeable or if evaluation technologies are primitive then $p$ will be close to $1 / 2$. If there is an a priori, theory-driven bias against $\mathrm{A}$, then $p$ could be less than $1 / 2$. In general $p$ is in $(0,1)$.

We assume that the impact of social influence is linearly increasing in the proportion of the "converted" who have adopted in a particular adoption. Thus, if $M_{t}$ denotes the number of people who by period $t$ have chosen $\mathrm{A}$ and $N_{t}$ denotes the number who have selected $\mathrm{B}$, then the social pressure to choose A is simply $M_{t} /\left(M_{t}+N_{t}\right)$. The social pressure to choose B is, of course, $N_{t} /\left(M_{t}+N_{t}\right) .{ }^{4}$ (We will use $m_{0}$ and $n_{0}$ to denote the initial number of adherents to $\mathrm{A}$ and $\mathrm{B}$, respectively.)

Since we are trying to construct a simple benchmark model that has a clean structure, we assume that an agent's choice is simply a weighted average of the

\footnotetext{
${ }^{3}$ We shall often interpret $\mathrm{A}$ and $\mathrm{B}$ as competing innovations, but the model allows for different interpretations. For example, one of the options could be the population's status quo alternative while the other is an innovation. We will return to this specific interpretation in Section 5

${ }^{4}$ This is consistent with a simple search process: if the decision maker looks for social cues (i.e., the choices that the already-converted have made), then with probability $M_{t} /\left(M_{t}+\right.$ $N_{t}$ ) the first convert she bumps into is an A-adherent. And so with that probability she is persuaded to adopt A (conditional on her choosing via social imitation).
} 
above components (individual judgement and social influence). Thus

$$
P[\text { agent in period } t+1 \text { chooses } A]=\alpha \cdot p+(1-\alpha) \frac{M_{t}}{M_{t}+N_{t}}
$$

where $0 \leq \alpha \leq 1$. Similarly, the probability that the chooser in period $t+1$ selects $B$ is

$$
P[\text { agent in period } t+1 \text { chooses } B]=\alpha \cdot(1-p)+(1-\alpha) \frac{N_{t}}{M_{t}+N_{t}} .
$$

Festinger's hypothesis - that people are more open to social influence when evidence is equivocal - amounts here to assuming that $\alpha$ and $p$ are positively correlated if $p>1 / 2$ and negatively correlated if $p<1 / 2$. We believe that this is a sensible proposition but we do not require it for our analytical results. We do use it in most of our simulations, however. Moreover, Festinger's hypothesis informs our understanding of what we consider soft technologies: in the context of Eq. (11), a soft technology is one with a $p$ in the vicinity of $1 / 2$ and a low $\alpha$.

To get a feel for how this process works it is useful to consider first the two extreme cases: i.e., when $\alpha=1$ and $\alpha=0$. The former is simply an independent trials process with a probability of "heads" of $p$. This process and its properties are well-understood. The case of $\alpha=0$ (pure social influence) is the standard Polya's urn process [PE23. Given that we are particularly interested in soft technologies, which here are represented by low values of $\alpha$, we will pause for a moment to recapitulate its features.

\section{Pure social influence $(\alpha=0)$}

Suppose 100 people have made up their minds, with 70 having chosen A and 30 having chosen B. Then in the current period agent 101 has a $70 \%$ chance of selecting A. Consequently the expected proportion of the population who cleave to $\mathrm{A}$ is $.7\left(\frac{71}{101}\right)+.3\left(\frac{70}{101}\right)=\frac{1}{101}(.7 \cdot 71+.3 \cdot 70)=\frac{1}{101}(70.7)=.70$; i.e., the expected proportion exactly equals the current proportion. It is easy to show that this martingale property holds in general: on average the pure social influence process stays exactly where it currently is. That is,

$$
E\left[F_{t+k} \mid F_{t}=\frac{M_{t}}{M_{t}+N_{t}}\right]=\frac{M_{t}}{M_{t}+N_{t}}=F_{t}
$$

for all $k>0$. Hence the pure social influence process is strongly path dependent: in expectation it tends to stay wherever it is - i.e., wherever it has arrived via the particular sample path it has been traveling.

Proposition 1. If $m_{0}=n_{0}=1$ (and $\left.\alpha=0\right)$, then $P\left[M_{t}=1\right]=P\left[M_{t}=2\right]=$ $\cdots=P\left[M_{t}=t+1\right]=1 /(t+1)$, for all $t>0$.

Proof. This is a classical result PE23]. 


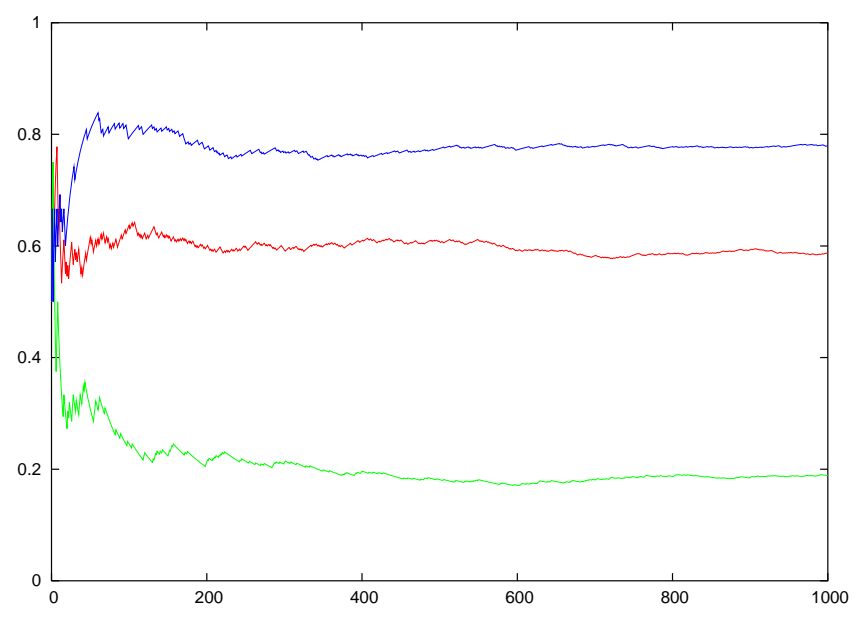

Figure 1: A simulation of the pure Polya's urn process for 1000 rounds. The initial condition is one A and one B. As can be seen from the figure, the three sample paths converge to different limits.

Thus every feasible outcome is equally likely at every date: given a fair start, the pure process of linear social pressure is completely 'blind'. Therefore the process is just as likely to wind up generating a heterogeneous diffusion, with half the population championing $\mathrm{A}$ and the other half $\mathrm{B}$, as it is to wind up with a sharply skewed outcome, with nearly everyone backing A. ${ }^{5}$

It is instructive to look at a typical sample path; see Fig. 1 Note that initially the relative frequencies of A- versus B-adherents swing wildly: with only a small number of initial converts, early adopters have a lot of influence. But once hundreds of people have taken sides subsequent adopters have little impact on relative frequencies, and so the process settles down. Hence it may appear to agents inside the process that the diffusion is moving toward some predetermined equilibrium. Part of this impression is correct: as $t \rightarrow \infty$, any sample path of this process will settle down near some long-run proportion of A-adherents and B-adherents. But we know from Proposition 1 that this asymptotic state was not at all predetermined: ex ante, every outcome is just as (un)likely as every other.

What does the pure Polya process tell us about the complex process, in which objective evidence does play a role? Recall that by Festinger's hypothesis, soft technologies have low $\alpha$ 's. Moreover, an agent's adoption probability, as represented by Eq. (1), is continuous in $\alpha$. Hence for $\alpha$ "close" to zero the

\footnotetext{
${ }^{5}$ It is worth mentioning that Proposition 1 depends upon the initial seed being $m_{0}=$ $n_{0}=1$. If it is a fair start but there are more two initial champions then the resulting distributions are not uniform, though they are symmetric around $1 / 2$. Nevertheless, the martingale conditional expectations property does continue to hold, for any values of $m_{0}$ and $n_{0}$. So in this sense the strong path dependence property is insensitive to the initial conditions.
} 
diffusion will approximate a pure social influence process: it will be "nearly" blind, ex ante.

In such circumstances agents are influenced by a mixture of conformity and individual judgment about the evidence. Yet they may not have good access to the fine structure of their own choice processes WB98. For one thing, they may not recognize how much of their choice is influenced by social cues. And even if they do, they may rationalize that part of it, along the lines of herding models: the behavior of peers conveys information, and so it is rational to be socially influenced. And of course that may be so. But if $\alpha$ is sufficiently low there is a good chance (less than half but still appreciable) that at date $t$ a majority of the converts will back the weaker option; hence the next adopter could be led astray.

Further, we suspect that people involved in a diffusion of a soft technology do not have good intuitions for the stochastic properties of the process. In particular, we suspect that the fact that with a low $\alpha$ a soft technology could, given a large population of adopters, wind up at many different outcomes with nearly equal ex ante probabilities is underappreciated. Life unfolds as a sample path, and the "settling down" feature of the particular sample path one inhabits (as in Fig. 1) will be much more salient than theoretical ex ante probabilities - if the latter are recognized at all.

\section{Properties of the Complex Process: $\alpha \in(0,1)$}

\subsection{The mean of the process}

We now directly investigate properties of the complex process. First let us examine how it behaves over time.

Proposition 2. Assume $\alpha>0$. EF $\rightarrow p$ monotonously as $t \rightarrow \infty$.

In fact, instead of expectations, we can establish a strong convergence result:

Proposition 3. $F_{t} \rightarrow p$ a.s. as $t \rightarrow \infty$.

Thus the process exhibits a constant drift or bias toward $p$, the value of individual judgment. This reason behind this is because the martingale property is broken. In fact, instead of Eq. (3), it can be shown (see Appendix) that for the complex process $\alpha \in(0,1)$,

$$
E\left[F_{t+1} \mid F_{t}\right]=F_{t}+\frac{\alpha\left(p-F_{t}\right)}{t+s+1}
$$

Note that the process could be mostly one of social construction-most of the weight is on imitation - yet improvement will tend to occur anyway, if $\frac{m_{0}}{m_{0}+n_{0}}<$ $p$. Henceforth we denote $\frac{m_{0}}{m_{0}+n_{0}}$ by $f_{0}$.

Next let us consider how $F_{t}$ is affected by variations in the model's two basic parameters, $\alpha$ and $p$. The latter's effect is both obvious and unconditional. Clearly, the average fraction of correct choices, $E F_{t}$, is increasing in $p$. But more can be said. 

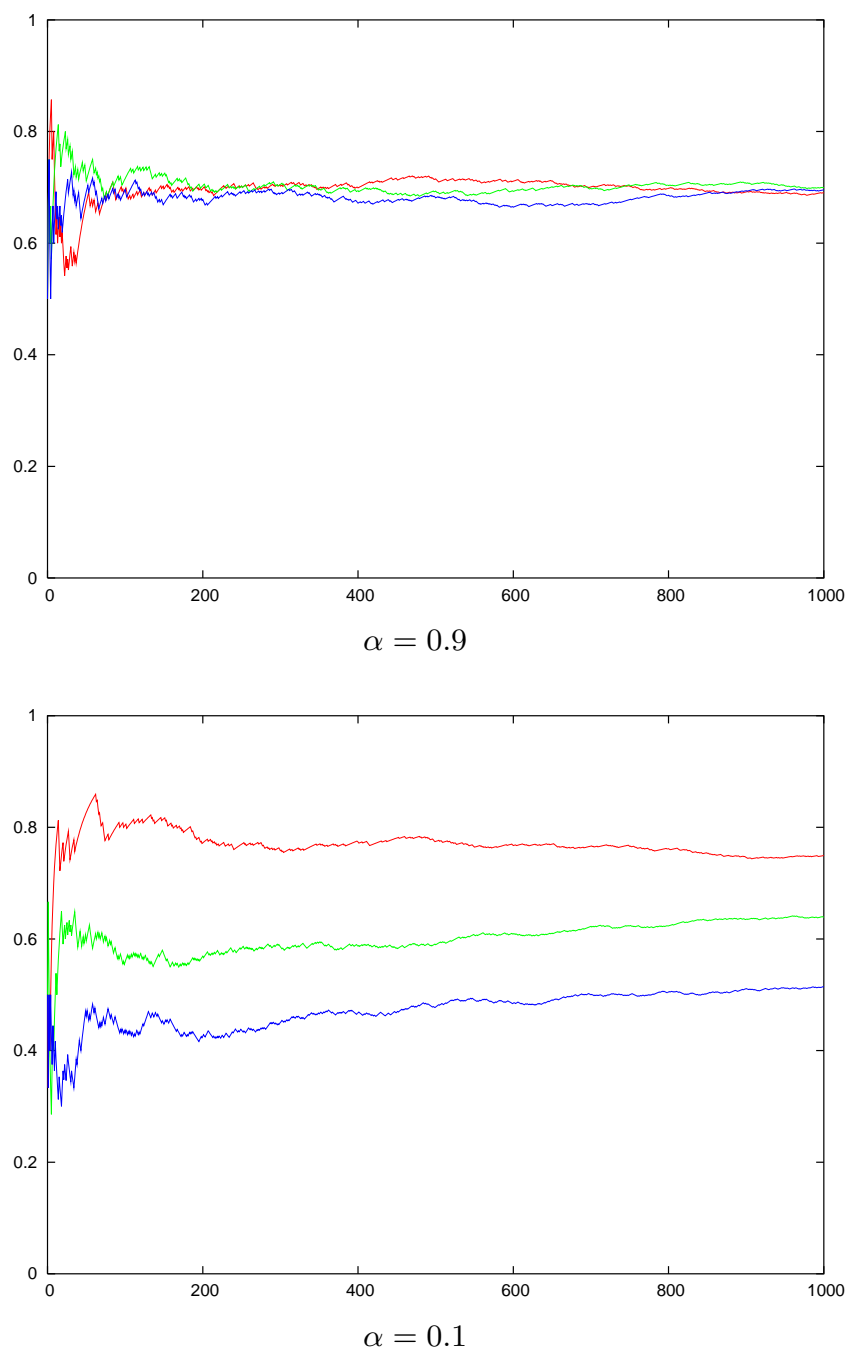

Figure 2: Simulations of the complex process after 1000 rounds. In both cases ( $\alpha=0.9$ and 0.1 ) we choose $p=0.7$ and initial condition one $\mathrm{A}$ and one $\mathrm{B}$. In each figure three sample paths are shown. As can be seen, the convergence rate for small $\alpha$ is much slower than the rate for large $\alpha$. 
Proposition 4. A higher value of $p$ yields a distribution of $F_{t}$ that stochastically dominates a distribution of $F_{t}$ produced by a lower value of $p$, for all $t>0$.

This immediately implies that $E F_{t}$ is increasing in $p .^{6}$

It is worth emphasizing that this strong effect obtains even if the process is mostly one of social construction (low $\alpha$ 's). Thus the presence of social construction does not utterly prevent "rational engineering" (e.g., concerning the technology of evaluation) from having benign effects. (Of course, in a sense this is built into the model, but hopefully it is built-in in a plausible way.)

Further, Festinger's hypothesis implies that if $p>1 / 2$, then as the evaluation technology improves, decision makers will rely more on the evidence and less on social cues: the rise in $p$ will be followed by an increase in $\alpha$. This indirect impact of changes of $p$ might be just as important as its direct effect on $E F_{t}$. This naturally raises the question, how do changes in $\alpha$ affect the process?

\section{Proposition 5.}

(i) If $f_{0}<p$, then $E F_{t}$ is increasing in $\alpha$, for all $t>0$.

(ii) If $f_{0}>p$, then $E F_{t}$ is decreasing in $\alpha$, for all $t>0$.

Thus, absent a lucky start $\left(f_{0}>p\right)$, less reliance on social cues improves matters. Consequently, if Festinger's hypothesis is correct, improvements in evaluation technology have two benign effects on diffusions that don't enjoy lucky starts: the obvious direct one (Proposition 4) and a less obvious indirect one (Proposition 5), via increases in $\alpha$.

\subsection{The unpredictability of the process}

If we compare the two pure processes, it is evident that, given a standard start $\left(m_{0}=n_{0}=1\right)$, the pure social process is less predictable (as measured by the variance of $F_{t}$ ). When the diffusion is purely a matter of social construction, every feasible outcome is equally likely; this is intuitively very random, and it translates into one with a high variance. In contrast, even the variant of the pure individualistic process with maximal variance $(p=1 / 2)$, extreme outcomes - nearly everyone choosing A or B, for example - are less likely than other outcomes. So the variance must be less than that produced by pure social imitation.

Since the complex process is, at the level of individual choice, a weighted average of the two pure processes, it is intuitively reasonable that the variance of the complex process would be increasing in $1-\alpha$, the weight on the higher variance component, given a fair start. This intuition is correct, but since it conditions on a particular starting point it is incomplete. The next result shows that more weight on the social component can reduce the variance of the complex process, at least for awhile.

\footnotetext{
${ }^{6}$ However, the converse does not hold: an increase in the mean fraction of correct choices does not imply that one distribution stochastically dominates the other. So Proposition 4 is stronger than just a comparison of means.
} 
Proposition 6. $\partial\left(\operatorname{var} F_{t}\right) / \partial \alpha<0$ for all $t>0$ iff either

(i) $f_{0}<p$ and $p_{0}=(1-\alpha) f_{0}+\alpha p>1 / 2$, or

(ii) $f_{0}>p$ and $p_{0}=(1-\alpha) f_{0}+\alpha p<1 / 2$.

Thus for parameters in this range, increases in the weight on evidence both improve community-wide accuracy (on average) and reduce the variance of outcomes.

While it makes sense that the more socially constructed a diffusion is the more variable are its outcomes, it is important to emphasize that this conclusion does not always hold, as is indicated by the "only if" part of Proposition [6 Here is why. Suppose initially A-adherents are few and far between; for simplicity, let $f_{0}=0$. Then if diffusion were based only on imitation $(\alpha=0)$, everyone would adopt the wrong option - and the process, although built completely on social cues, would exhibit no variability whatsoever. In such a case small increases in $\alpha$ would increase the variance of the outcomes, at least in the early goings. Of course this increase in variance is thoroughly benign: without it, the diffusion would be stuck in a highly predictable but consistently inferior sample path.

\section{Proposition 7.}

(i) $\partial\left(\operatorname{var} F_{t}\right) / \partial p<0$ for all $t>0$ if $p \geq 1 / 2$ and $p_{0}>1 / 2$;

(ii) $\partial\left(\operatorname{var} F_{t}\right) / \partial p>0$ for all $t>0$ if $p \leq 1 / 2$ and $p_{0}<1 / 2$.

Regarding part (ii), note that increases in $p$ increase the variance in a socially desirable way. However, people who like predictability could resent the "rationalizing" effect of improvements in program information and evaluation technology, because in these circumstances increasing $p$ makes things "messier" and less predictable.

As a matter of interpretation, one might say that a $p \leq 1 / 2$ and an $p_{0}<1 / 2$ involves a "soft" technology with a vengeance: there is some kind of bias against the better technology, maybe because it is not as trendy, and there is also a bias in terms of the initial social proclivities $\left(p_{0}<1 / 2\right)$. In this case, improving evaluation technology also creates a "messier" and more confusing process ex ante, in that such improvements increase the variance of the outcomes.

Indeed, even if $p>1 / 2$ (i.e., the "standard" case), we know that in period 1 the variance of $F_{1}$ is increasing in $p$ if $p_{0}<1 / 2$. (And probably this will hold for some finite number of periods after period 1 too.) This is a very natural case of a soft technology: there is a social bias against the better technology (bad luck, essentially, or maybe glamour is on the side of the weaker technology), in that $f_{0}<1 / 2$, and things are noisy enough so that $p$, though above $1 / 2$, is still fairly low. Hence if Festinger's hypothesis were to kick in, so that $\alpha$ were pretty low too, then $p_{0}<1 / 2$, and the variance of $F_{1}$ would be increasing in $p$.

\subsection{The effects of initial conditions}

There are two types of initial conditions: the size of the initial seed $\left(m_{0}+n_{0}\right)$ and the proportion $\left(f_{0}=\frac{m_{0}}{m_{0}+n_{0}}\right)$. Each has effects independently of the other 
(i.e., holding the other fixed), so we will examine them separately and in a ceteris paribus manner.

\subsubsection{Varying the size of the initial seed}

Proposition 8. Suppose $F_{t}^{\prime}$ is a bigger process than $F_{t}$ in that $m_{0}^{\prime}=k m_{0}$ and $n_{0}^{\prime}=k n_{0}$, where $k$ is an integer larger than one. In all other respects the two processes are identical.

(i) $E F_{t}^{\prime}<E F_{t}$ for all $t>0$ iff $f_{0}<p$.

(ii) $E F_{t}^{\prime}>E F_{t}$ for all $t>0$ iff $f_{0}>p$.

Thus a bigger initial seed acts as an inertial anchor, slowing down the movement of $E\left[F_{t}\right]$ to its attractor $p$.

\subsubsection{Varying the initial proportions}

It is obvious that, all else equal, increasing the initial bias toward A (i.e., increasing $f_{0}$ ) boosts $E F_{t}$ at every date. Suppose, e.g., we start out with $m_{0}+1$ A-converts, instead of with just $m_{0}$. This increases the social conformity pressure for the agent in period 1 to adopt $\mathrm{A}$. That in turn increases $F_{1}$, the expected fraction of the converts who adhere to $\mathrm{A}$ at the end of period 1 , which in turn provides more social cues to the decision maker in period 2 to adopt $\mathrm{A}$ and so on.

But a higher $f_{0}$ has an even stronger effect-stronger even than ordinary first-order stochastic dominance. To see what this sense is, consider the following definition.

Definition 1. Suppose the support of $F_{t}$ and $F_{t}^{\prime}$ can be divided into three mutually disjoint subsets: a non-empty set of high states $\left\{f_{h_{1}, t}, f_{h_{2}, t}, \ldots, f_{h_{a}, t}\right\}, a$ non-empty set of low states $\left\{f_{l_{1}, t}, f_{l_{2}, t}, \ldots, f_{l_{b}, t}\right\}$, and a possibly empty set of intermediate states $\left\{f_{m_{1}, t}, f_{m_{2}, t}, \ldots, f_{m_{c}, t}\right\}$. Suppose the three subsets are connected in the sense that any low state is less than any intermediate state, and any intermediate state is less than any high state. We say that $F_{t}^{\prime}$ is stochastically bigger than $F_{t}$ in a strong sense if $P\left[F_{t}^{\prime}=f_{h, t}\right]>P\left[F_{t}=f_{h, t}\right]$ for any high state $h, P\left[F_{t}^{\prime}=f_{m, t}\right]=P\left[F_{t}=f_{m, t}\right]$ for any intermediate state $m$, and $P\left[F_{t}^{\prime}=f_{l, t}\right]<P\left[F_{t}=f_{l, t}\right]$ for any low state $l$.

Thus a distribution of $F_{t}$ that is stochastically bigger in a strong sense puts strictly more weight on high fractions of converts who have chosen correctly, and strictly less weight on low fractions. (Clearly this implies first-order stochastic dominance, but the converse need not hold, so this is in fact a "strong sense" of stochastic dominance.)

Proposition 9. All else equal, the distribution of $F_{t}$ is stochastically increasing, in a strong sense, in $f_{0}$, for all $t>0$. 
Naturally, smaller $f_{0}$ 's produce distributions of $F_{t}$ that are stochastically smaller, in a strong sense. Suppose, therefore, that A is an innovation that is superior to B. If B is not an innovation - it is in fact the status quo - and the community is quite traditional (everyone initially uses $\mathrm{B}$, though people are willing to consider an innovation), then effectively $f_{0}$ equals zero. This is the toughest possible starting point for an objectively superior innovation.

The effect of varying initial bias on the variance of the process is perhaps less intuitive.

Proposition 10. $\partial\left(\operatorname{var} F_{t}\right) / \partial f_{0}<0$ for all $t>0$ if $p_{0}>1 / 2$ and $p>1 / 2$.

\subsection{The convergence rate}

If we wish to use our complex process to explain diffusion of innovations in the real world, only studying the limit behavior is not enough. If the characteristic time needed to reach the asymptote state is too long to be observed, then the asymptote state cannot be very meaningful in the practical sense. A rough estimation of the convergence rate of the complex process can be achieved from a mean-field approximation. Neglecting the noise term, the stochastic process $F_{t}$ can be approximated by Eq. (4):

$$
F_{t+1}=F_{t}+\frac{\alpha\left(p-F_{t}\right)}{t+s+1} \text {. }
$$

Further approximating $F_{t}$ by a continuous process $F(t)$, we can write

$$
\frac{d F(t)}{d t}=\alpha \frac{p-F(t)}{t+s+1} \sim \alpha \frac{p-F(t)}{t} .
$$

Solving for $F(t)$, we find

$$
|F(t)-p| \sim t^{-\alpha}
$$

If we define a characteristic convergence time $T$ to be the time it takes $F(t)$ to converge to a vicinity within $\epsilon$ from $p$, then we have

$$
T \sim\left(\frac{1}{\epsilon}\right)^{\frac{1}{\alpha}} .
$$

Thus as $\alpha \rightarrow 0$, the characteristic convergence time diverges exponentially in $1 / \alpha$. Indeed, when $\alpha=0$ (Polya's urn) the process never converges to $p$. (To be precise, the Polya process can converge to any $p \in[0,1]$, but the probability that it converges to any particular $p$ is zero.)

The mean-field estimation is in principle only for the mean of $F_{t}$, of course. For a fine estimation of the variance of $F_{t}$, we need a central limit theorem.

\section{Proposition 11.}

(i) If $1 / 2<\alpha \leq 1$, then as $t \rightarrow \infty, \sqrt{t}\left(F_{t}-p\right)$ converges in distribution to a normal distribution with mean zero and variance $p(1-p) /(2 \alpha-1)$. In particular, $\operatorname{var} F_{t}=O(1 / t)$.

(ii) If $0<\alpha \leq 1 / 2$, then $\operatorname{var} F_{t} \rightarrow 0$ slower than $O(1 / t)$. 


\subsection{Implications regarding herding and massive confor- mity}

Assume, as in cascade models with rational agents, that the "initial seed" is exactly one person who chooses A with probability $p$ and $B$ with probability $1-p$, and everyone else follows sequentially.

Definition 2. We say that there is "herding" if after some period T everyone makes the same choice. ${ }^{7}$

Fact 1. Herding occurs in our model (with positive probability) iff one of the following condition holds:

(a) $\alpha>0$ and $p=1$; then we get herding on option $A$.

(b) $\alpha>0$ and $p=0$; then we get herding on option $B$.

(c) $\alpha=0$; then we get herding on option $A$ with probability $p$ and herding on option $B$ with the complementary probability $1-p$.

Proof. Sufficiency. All three parts are trivial, by induction.

Necessity. Suppose none of (a)-(c) hold. Then we know that $\alpha>0$ and $p \in$ $(0,1)$. By Prop. 3 $F_{t} \rightarrow p$ almost surely. Hence both A and B are chosen infinitely often with probability one.

Fact 2. Under the assumptions of our model, if heterogeneous behavior ever occurs then herding is impossible (with probability one).

Proof. By Fact 1, if heterogeneous behavior has emerged by some date $t>0$ then the extreme conditions (a)-(c) in Fact 1 cannot hold. Hence we know that $\alpha>0$ and $p \in(0,1)$. The rest is the same as the necessity part of Fact 1.

Conformity could be overwhelming - nearly everyone in a community winds up making the same choice - without being complete. One of the surprising results of the rational cascade models is that massive-conformity-in-the-making can be very fragile. As Bikhchandani, Hirshleifer and Welch put it, "A little bit of public information (or an unusual signal) can overturn a long-standing informational cascade. That is, even though a million people may have chosen one action, seemingly little information can induce the next million people to choose the opposite action. Fragility is an integral component of the informational cascades theory!" ([BHW96] original emphasis).

But this fragility is intimately linked to the agents' complete rationality and deep understanding of informational cascades. As Bikchandani et al. remark, in standard rational models "everyone knows that there is very little information in a cascade BHW96. That "everyone" pertains, of course, to the model's rational agents, not to real decision makers: the evidence is that the latter do

\footnotetext{
${ }^{7}$ This is the term some scholars (e.g., Ban92) use. Others (e.g., BHW96) call this an informational cascade.
} 


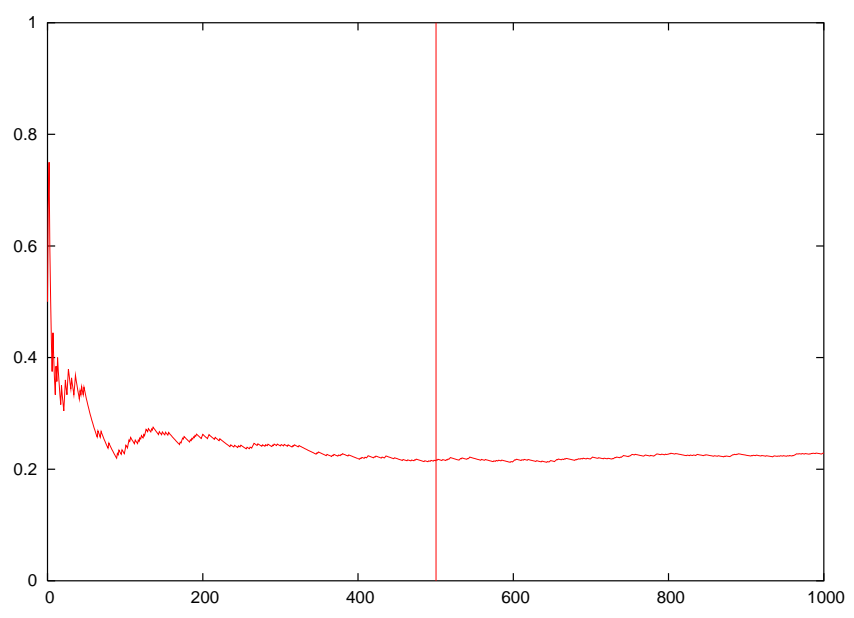

Figure 3: A simulation of the complex process with $\alpha=0.5$ and initial seed one A and one B. $p$ is set to 0.2 for the first 500 rounds and is updated to 0.3 for another 500 rounds. The vertical line indicates the change point. As can be seen, the sample path is not significantly affected by the new level of $p$.

not realize that there is very little information in a cascade. ${ }^{8}$ The agents in our model are imperfectly rational and lack a deep understanding of cascades. Hence, diffusion processes in our model are not fragile (in the above sense). This is easily established by re-inspecting Eq. (1): the probability that an agent makes the correct decision is continuous in $p$, so a little bit of new public informationrepresented as a sudden positive shock to $p$-will only increase the probability of choosing correctly by a little bit. (For an illustration of this, see Fig. [3])

\section{$5 \quad$ Alternative Interpretations}

It is worth noting that one can use the model to represent situations in which there is a status quo option that everyone already uses. Then the diffusion is simply this: in every period one agent has an opportunity to either take up the innovation (say, A) or keep the status quo (say, B). Further, one could allow for the innovation to be objectively inferior to the status quo.

Under this interpretation of a novel technology competing with a status quo, it is reasonable to suppose that $f_{0}$ equals zero. This is, from the point of view of an innovation, the toughest possible starting point.

If it's a soft innovation then presumably both $p$ and $\alpha$ are relatively low, so it is probably the case that $\alpha p+(1-\alpha) f_{0}$ is less than one-half. Hence by

\footnotetext{
${ }^{8}$ This is the pattern that Kubler and Weiszacker found in their experiments on cascades KW04. As they put it, "players do not consider what their predecessors thought about their respective predecessors. Thus, they do not understand that some of the decisions they observe have been herding decisions, not based on any private information (pp. 438).
} 
Proposition [6] decreasing the reliance on social cues (i.e., a smaller $1-\alpha$ ) would increase the variance of outcomes.

\section{Extensions of the Model}

\subsection{Endogenizing Festinger's Hypothesis}

If Festinger's hypothesis is correct, then the weight on social cues, should depend on how difficult the choice is. That is, $\alpha$ should be a function of $p$. We can stipulate a priori several properties that this function should have. First, $1-\alpha$ should reach its maximum when $p=1 / 2$ : it is in this circumstance that an agent is maximally uncertain about the relative merits of A versus B and so, following Festinger, s/he would be maximally reliant on social cues. Second, as a benchmark the function should be symmetric around one half: $f(.5-k)=$ $f(.5+k)$, for $.5 \leq k \leq 1$.

A simple function with both properties is $1-\alpha=4 p(1-p)$. Then the probability that the chooser in period $t$ would pick A would equal $4 p(1-p)$. $F_{t}+1-4 p(1-p) \cdot p$.

An interesting feature of this choice equation is that it creates the possibility that the probability of a correct choice is decreasing in $p$. This seems bizarre but it is explicable: it arises because $\alpha$ is a function of $p$. Note that $4 p(1-p)$, the weight on social cues, is increasing when $p$ is less than one half, since in this range increases in individual-level accuracy make the choice problem more confusing or troubling. Thus, as we suggested earlier, increases in $p$ have two effects: a direct effect on individualistic choice (which is always benign, as shown by Proposition 4) and an indirect one on the weights (which is not always benign). For certain parametric values the indirect effect is sufficiently large and negative so as to swamp the positive direct effect. ${ }^{9}$

Thus endogenizing Festinger's hypothesis will generate interesting hypotheses.

\subsection{Diffusion in Communities with Internal Structure}

\subsubsection{Status Hierarchies}

There is considerable empirical support for the hypothesis that the higher the decision maker's status, the more impact his/her adoption choice has on the unconverted (e.g., SS98, pp. 275]). It would be easy to incorporate this empirical regularity in a stark way in the current model: only high status agents are imitated; the adoption choices of low status agents are ignored.

\footnotetext{
${ }^{9}$ To see this concretely, note that the derivative of $4 p(1-p) \cdot f_{0}+1-4 p(1-p) \cdot p$ with respect to $p$ equals $4(1-2 p) \cdot f_{0}+\left(1-8 p+12 p^{2}\right)$. Then suppose for example that $f_{0}=0$ and $p=.25$. With these parametric values the derivative equals -.25 , so small increases in $p$ make the agent in period one more likely to err. However, if $p$ is sufficiently big-specifically, if it exceeds $\max \left(.5, f_{0}\right)$ - then increases in $p$ do decrease the chance of mistakes. (When $p>.5$ then a higher $p$ decreases the decision maker's confusion, thus raising the weight on individualistic judgment, which is benign since $p>f_{0}$.)
} 
An intriguing issue concerns the relative competence of high status and low status agents. In diffusions that tap into a relatively strong scientific or technical base (pharmaceuticals, computer hardware, etc.), one would expect higher status agents to be more likely to make the right choice (higher $p$ 's). However, there may be circumstances in which status is purely a subjective phenomenon, lacking any objective correlate. (People inside the community may believe that higher status is correlated with more expertise but this would be an illusion.)

\subsubsection{Clustered Interaction}

Most diffusions occurs in communities that exhibit biased interactions. In general, executives in the same industry are more likely to interact with each other than with executives in a different industry. School superintendents in the same state are more likely to encounter each other than are superintendents in different states. (At the limit-if these subcommunities were completely sealed off from each other - then our model applies as it is to each subcommunity.) ${ }^{10}$

\subsection{Leakage of Information}

Here, $p$ would be a function of time. Typically we would expect that $p$ would increase over time, as information about the technology (by the already-converted or by third parties) leaks out. As already noted, the baseline diffusion process (stationary $p$ ) is robust: small positive shocks to $p$ will on average have small effects on the fraction of the population that adopts option A.

\subsection{Waves of Innovations}

Today it seems that the business world never rests. No sooner has one innovation passed from the scene - or at least from public attention - than another one appears. Becauase there are good reasons for this - it is not accidental Abr96 one can expect this pattern to continue indefinitely.

Although waves of innovation will obviously produce some new patterns, we suspect that some of the present paper's results will continue to hold. In particular, we conjecture that if the waves are composed of soft technologies, with $p$ around $1 / 2$ and strong propensities to imitate, then the process will continue to be Polya-like in two senses. First, objectively uncertainty will be very great: many adoption patterns will be possible ex ante. Second, if the waves do not come too often then sample paths of diffusion will settle down. Hence, subjectively it will feel as if the process is moving toward a predetermined equilibrium - an illusion.

${ }^{10}$ For a study of the influence of social structure on opinion formation, see e.g. WH05 [SR05]. 


\section{Conclusion}

Because they diffuse with little objective information about their effects, soft technologies pose challenges for decision makers. Psychologists have long argued that when faced with such choice problems, people use a reasonable though imperfect imitation heuristic. We have presented a mathematical model of diffusion that combines this heuristic with agents' efforts to make factually-grounded decisions and we established both analytically and computationally that such processes exhibit clear stochastic properties. We then showed that the dynamics of the model leads to outcomes that appear to be deterministic in spite of being governed by a stochastic process. In other words, when the objective evidence for the adoption of a soft-technology is weak, any sample path of this process quickly settles down to a fraction of adopters that is not predetermined by the initial conditions: ex ante, every outcome is just as (un)likely as every other. When the objective evidence is strong, the process settles down to a value that is determined by the joint effect of the quality of the evidence and the agents' competence. In neither case does the proportion of adopters settle into either zero or one: pure herding does not occur except in parametrically extreme situations.

Further, unlike informational cascades generated by fully rational actors, the process of the present model is robust: diffusions that have for a long time tilted massively toward one option cannot be suddenly derailed by small infusions of new public information. The fragility of cascades generated by fully Bayesian agents is, we believe, an artifact of unrealistic assumptions of hyper-rationality. Diffusions may be initially volatile, as they are in the present model, but we believe that these processes stabilize once the weight of public opinion has been brought to bear. ${ }^{11}$

\footnotetext{
${ }^{11}$ Of course, the introduction of a new innovation can, by restarting the process, destabilize it. But that is not what is producing fragility in the full-rationality cascades: these are not robust against small shocks associated with the pre-existing options. Moreover, as indicated earlier, we believe that the present model can be extended to accommodate waves of innovation.
} 


\section{References}

[Abr96] Eric Abrahamson. Management fashion. Academy of Management Review, 21:254-286, 1996.

[Ban92] A. V. Banerjee. A simple model of herd behavior. Quarterly J. Econ., 107:797-818, 1992.

[BHW92] S. Bikhchandani, D. Hirshleifer, and I. Welch. A theory of fads, fashion, custom, and cultural change as informational cascades. $J$. Pol. Econ., 100:992-1026, 1992.

[BHW96] Sushil Bikhchandani, David Hirshleifer, and Ivo Welch. Informational cascades and rational herding: An annotated bibliography. Working Paper: UCLA/Anderson and Ohio State University and Yale/SOM. http://welch.econ.brown.edu/cascades/, 1996.

[Blu54] J. R. Blum. Approximation methods which converge with probability one. Ann. Math. Statist., 25:382-386, 1954.

[Cam98] Colin Camerer. Bounded rationality in individual decision making. Experimental Economics, 1:163-183, 1998.

[Chu54] K. L. Chung. On a stochastic approximation method. Ann. Math. Statist., 25:463-483, 1954.

[Fes54] Leon Festinger. A theory of social comparison processes. Human Relations, 7:117-140, 1954.

[KW04] D. Kubler and G. Weiszacker. Limited depth of reasoning and failure of cascade formation in the laboratory. Review of Economic Studies, 71:425-441, 2004.

[Las98] Larry Lashway. Creating a learning organization. Eugene, OR: ERIC Clearinghouse on Educational Management, ED420897, 1998.

[MC04] Cleborne Maddux and Rhoda Cummings. Fad, fashion, and the weak role of theory and research in information technology in education. Journal of Technology and Teacher Education, 12:511-533, 2004.

[MW02] Michael Macy and Robert Willer. From factors to actors: Computational sociology and agent-based modeling. Annual Review Of Sociology, 28:143-160, 2002.

[PE23] G. Polya and F. Eggenberger. über die statistik verketteter vorgänge. Zeitschrift für Angewandte Mathematische Mechanik, 3:279-289, 1923.

[Pem01] Robin Pemantle. Random processes with reinforcement. Preprint, http://www . math. upenn.edu/ pemantle/reinforce/paper/statsci011005.ps 2001. 
[RM51] H. Robbins and S. Monro. A stochastic approximation method. Ann. Math. Stat., 22:400-407, 1951.

[SR05] V. Sood and S. Redner. Voter model and heterogeneous graphs. Phys. Rev. Lett., 94:178701, 2005.

[SS98] D. Strang and S. Soule. Diffusion in organizations and social movements: From hybrid corn to poison pills. Annual Review of Sociology, 24:265-290, 1998.

[WB98] D. Wegner and J. Bargh. Control and automaticity in social life. In D. Gilbert, S. Fiske, and G. Lindzey, editors, The Handbook of Social Psychology. McGraw-Hill, Boston, 1998.

[Wel92] I. Welch. Sequential sales, learning, and cascades. J. Finance, 47:695$732,1992$.

[WH05] Fang $\mathrm{Wu}$ and Bernardo A. Huberman. Social structure and opinion formation. http://http://www.hpl.hp.com/research/idl/papers/opinions/opinions.pdf 2005 . 


\section{Appendix}

Consider the following variation of Polya's urn. There are two types of alternatives, A and B. At time $t=0$ there are $s$ agents who have already made their choices, of which $m_{0}$ have chosen A and $n_{0}$ have chosen B. Denote the initial fraction of A-adherents to be $f_{0}=m_{0} / s$. Define the dynamics recursively as follows. Let $\left(M_{t}, N_{t}\right)$ be the (random) number of agents who have chosen A by the end of period $t$. In period $t+1$, an agent chooses $\mathrm{A}$ with probability

$$
P_{t}=\alpha p+(1-\alpha) \frac{M_{t}}{M_{t}+N_{t}},
$$

where $\alpha, p \in[0,1]$, and chooses $\mathrm{B}$ with probability $1-P_{t}$. If $\alpha=0$ this becomes a standard Polya's urn process. In the rest of this appendix we will assume $\alpha>0$.

We are interested in studying the (random) fraction of agents who have chosen A up to period $t$ :

$$
F_{t}=\frac{M_{t}}{M_{t}+N_{t}}
$$

Conditional on the information at time $t$, with probability $P_{t}$,

$$
\Delta F_{t+1}=F_{t+1}-F_{t}=\frac{M_{t}+1}{M_{t}+N_{t}+1}-\frac{M_{t}}{M_{t}+N_{t}}=\frac{1-F_{t}}{t+s+1},
$$

while with probability $1-P_{t}$,

$$
\Delta F_{t+1}=\frac{M_{t}}{M_{t}+N_{t}+1}-\frac{M_{t}}{M_{t}+N_{t}}=\frac{-F_{t}}{t+s+1} .
$$

Hence

$$
E_{t}\left[\Delta F_{t+1}\right]=\frac{P_{t}-F_{t}}{t+s+1}=\frac{\alpha\left(p-F_{t}\right)}{t+s+1} .
$$

Separating out the mean term, we can write

$$
\Delta F_{t+1}=\frac{\alpha\left(p-F_{t}\right)}{t+s+1}+\frac{X_{t+1}}{t+s+1}
$$

where $X_{t}$ is a martingale difference with the conditional distribution

$$
X_{t+1}= \begin{cases}1-P_{t} & \text { with probability } P_{t}, \\ -P_{t} & \text { with probability } 1-P_{t} .\end{cases}
$$

Eq. (14) falls into the general class of stochastic approximation processes that have been studied intensively in the statistics literature RM51, Pem01.

In what follows it is convenient to introduce an auxiliary random variable

$$
G_{t}=F_{t}-p
$$

so that Eq. (14) can be written as

$$
\Delta G_{t+1}=\frac{-\alpha G_{t}+X_{t+1}}{t+s+1}
$$


Proposition 2. $E F_{t} \rightarrow p$ monotonously as $t \rightarrow \infty$.

Proof. From Eq. (17) we have

$$
G_{t+1}=\left(1-\frac{\alpha}{t+s+1}\right) G_{t}+\frac{X_{t+1}}{t+s+1} .
$$

Taking expectation of both sides, we have

$$
E G_{t+1}=\left(1-\frac{\alpha}{t+s+1}\right) E G_{t}=\prod_{k=0}^{t}\left(1-\frac{\alpha}{k+s+1}\right) g_{0} .
$$

Because

$$
\sum_{k=0}^{\infty} \frac{\alpha}{k+s+1}=\infty
$$

the infinite product in Eq. (19) converges to zero monotonously. Thus $E G_{t} \rightarrow 0$ monotonously, or $E F_{t} \rightarrow p$ monotonously.

Lemma 1. Assume $\alpha>0$. If

$$
y_{n}=x_{n}+\alpha \sum_{k=1}^{n-1} \frac{x_{k}}{k}
$$

converges, then $x_{n} \rightarrow 0$.

Proof. Suppose $x_{n}$ does not converge to 0 . Then without loss of generality we can assume that $x_{n}>\epsilon>0$ infinitely often. It must also be that $x_{n}<\epsilon / 2$ infinitely often, otherwise we would have $x_{n} \geq \epsilon / 2$ eventually and $y_{n}$ would diverge. Because $y_{n}$ is Cauchy, we can find $N$ such that $\left|y_{m}-y_{n}\right|<\epsilon / 4$ for all $m, n \geq N$. Pick $n^{\prime} \geq \max \{N, 2 \alpha\}$ such that $x_{n^{\prime}}<\epsilon / 2$. Pick $m>n^{\prime}$ such that $x_{m}>\epsilon$. Let $n$ be the largest integer such that $n^{\prime} \leq n<m$ and $x_{n}<\epsilon / 2$. It is clear that $m, n$ chosen this way satisfy the following conditions:

$$
\left\{\begin{array}{l}
m>n \geq N \\
x_{n}<\epsilon / 2, \quad x_{m}>\epsilon \\
n<i<m \Rightarrow x_{i} \geq \epsilon / 2>0 .
\end{array}\right.
$$

Now we have

$$
\begin{aligned}
x_{m}-x_{n}=y_{m}-y_{n}-\alpha & \sum_{k=n}^{m-1} \frac{x_{k}}{k} \leq y_{m}-y_{n}-\alpha \frac{x_{n}}{n} \\
& <y_{m}-y_{n}+\frac{\alpha}{n}\left(x_{m}-x_{n}\right)<\frac{\epsilon}{4}+\frac{1}{2}\left(x_{m}-x_{n}\right) .
\end{aligned}
$$

Hence $x_{m}-x_{n}<\epsilon / 2$. But this contradicts with the fact that $x_{n}<\epsilon / 2$ and $x_{m}>\epsilon$. 
Proposition 3. $F_{t} \rightarrow p$ a.s. as $t \rightarrow \infty$.

Proof. Replacing $t$ by $k$ and summing up $k=0, \ldots, t-1$ in Eq. (17), we find a martingale:

$$
\tilde{G}_{t}=G_{t}-g_{0}+\sum_{k=0}^{t-1} \frac{\alpha G_{k}}{k+s+1}=\sum_{k=0}^{t-1} \frac{X_{k+1}}{k+s+1} .
$$

Furthermore, this martingale is nonnegative. The martingale convergence theorem thus ensures that $\tilde{G}_{t}$ converges to a (random) limit $\tilde{G}_{\infty}$ with probability 1. From Eq. (24) and Lemma the almost sure convergence of $\tilde{G}_{t}$ implies that $G_{t} \rightarrow 0$ almost surely, or $F_{t} \rightarrow p$ almost surely.

Proposition 4. A higher value of $p$ yields a distribution of $F_{t}$ that stochastically dominates a distribution of $F_{t}$ produced by a lower value of $p$, for all $t>0$.

Proof. Suppose $p^{\prime}>p$. It suffices to show by induction that for all $t, M_{t}^{\prime}$ stochastically dominates $M_{t}$, or $P\left[M_{t}^{\prime}>m\right] \geq P\left[M_{t}>m\right]$ for all $m \in \mathbb{N}$. The statement is correct for $t=0$ because $m_{0}^{\prime}=m_{0}$. Suppose the statement is correct for $t$, we prove for $t+1$.

Case 1. $\quad P\left[M_{t}^{\prime}=m\right] \geq P\left[M_{t}=m\right]$.

$$
\begin{aligned}
& P\left[M_{t+1}^{\prime}>m\right] \\
= & P\left[M_{t}^{\prime}>m\right]+P\left[M_{t}^{\prime}=m\right]\left[\alpha p^{\prime}+(1-\alpha) \frac{m}{t+s}\right] \\
\geq & P\left[M_{t}>m\right]+P\left[M_{t}=m\right]\left[\alpha p+(1-\alpha) \frac{m}{t+s}\right] \\
= & P\left[M_{t}>m\right] .
\end{aligned}
$$

Case 2. $\quad P\left[M_{t}^{\prime}=m\right]<P\left[M_{t}=m\right]$.

$$
\begin{aligned}
& P\left[M_{t+1}^{\prime} \leq m\right] \\
= & P\left[M_{t}^{\prime}<m\right]+P\left[M_{t}^{\prime}=m\right]\left[\alpha\left(1-p^{\prime}\right)+(1-\alpha)\left(1-\frac{m}{t+s}\right)\right] \\
\leq & P\left[M_{t}<m\right]+P\left[M_{t}=m\right]\left[\alpha(1-p)+(1-\alpha)\left(1-\frac{m}{t+s}\right)\right] \\
= & P\left[M_{t} \leq m\right] .
\end{aligned}
$$

\section{Proposition 5.}

(i) If $f_{0}<p$, then $E F_{t}$ is increasing in $\alpha$, for all $t>0$.

(ii) If $f_{0}>p$, then $E F_{t}$ is decreasing in $\alpha$, for all $t>0$.

Proof. See Eq. (19). 
Proposition 6. $\partial\left(\operatorname{var} F_{t}\right) / \partial \alpha<0$ for all $t>0$ iff either

(i) $f_{0}<p$ and $p_{0}=(1-\alpha) f_{0}+\alpha p>1 / 2$, or

(ii) $f_{0}>p$ and $p_{0}=(1-\alpha) f_{0}+\alpha p<1 / 2$.

Proof. "If" part. Taking variance of both sides of Eq. (18), we have

$$
\operatorname{var} G_{t+1}=\left(1-\frac{\alpha}{t+s+1}\right)^{2} \operatorname{var} G_{t}+\frac{1}{(t+s+1)^{2}} \operatorname{var} X_{t+1} .
$$

The variance of $X_{t+1}$ can be calculated as follows. First note that

$$
P_{t}=\alpha p+(1-\alpha) F_{t}=p+(1-\alpha) G_{t} .
$$

Then we can write

$$
\begin{aligned}
\operatorname{var} X_{t+1} & =E\left[X_{t+1}^{2}\right]=E\left[E_{t}\left[X_{t+1}^{2}\right]\right]=E\left[P_{t}\left(1-P_{t}\right)\right] \\
& =E P_{t}\left(1-E P_{t}\right)-\operatorname{var} P_{t} \\
& =E P_{t}\left(1-E P_{t}\right)-(1-\alpha)^{2} \operatorname{var} G_{t} .
\end{aligned}
$$

Plugging this back into Eq. (27), we obtain a recursive relation for $\operatorname{var} G_{t}$ :

$$
\operatorname{var} G_{t+1}=\frac{(t+s+2-2 \alpha)(t+s)}{(t+s+1)^{2}} \operatorname{var} G_{t}+\frac{E P_{t}\left(1-E P_{t}\right)}{(t+s+1)^{2}},
$$

where

$$
E P_{t}=p+(1-\alpha) E G_{t} .
$$

It is clear from this recursive relation that the conclusion holds if

$$
\frac{\partial\left(E P_{t}\left(1-E P_{t}\right)\right)}{\partial \alpha}<0 .
$$

Case (i) By hypothesis $g_{0}<0$. From Eq. (19) we see that $E G_{t}$ (being a negative sequence) is increasing in both $t$ and $\alpha$, so is $E P_{t}$ by Eq. (31). Therefore $E P_{t}>p_{0}>1 / 2$. Note that the function $f(x)=x(1-x)$ is decreasing in $x$ when $x>1 / 2$. Thus $E P_{t}\left(1-E P_{t}\right)$ is decreasing in $\alpha$ for all $t$.

Case (ii) Similar to (i).

"Only if" part. Letting $t=0$ in Eq. (30), we have

$$
\operatorname{var} G_{1}=\frac{p_{0}\left(1-p_{0}\right)}{(s+1)^{2}} .
$$

Taking partial derivative with respect to $\alpha$, we obtain

$$
\frac{\partial\left(\operatorname{var} G_{1}\right)}{\partial \alpha}=\frac{\left(f_{0}-p\right)\left(2 p_{0}-1\right)}{(s+1)^{2}} .
$$

Thus, in order to have $\partial\left(\operatorname{var} F_{1}\right) / \partial \alpha<0$, we must have $\left(f_{0}-p\right)\left(2 p_{0}-1\right)<0$, i.e., either (i) or (ii). 


\section{Proposition 7.}

(i) $\partial\left(\operatorname{var} F_{t}\right) / \partial p<0$ for all $t>0$ if $p \geq 1 / 2$ and $p_{0}>1 / 2$;

(ii) $\partial\left(\operatorname{var} F_{t}\right) / \partial p>0$ for all $t>0$ if $p \leq 1 / 2$ and $p_{0}<1 / 2$.

Proof. We see from Eq. (31) and Eq. (19) that

$$
\frac{\partial\left(E P_{t}\right)}{\partial p}=1+(1-\alpha) \frac{\partial\left(E G_{t}\right)}{\partial p}=1-(1-\alpha) \prod_{k=0}^{t-1}\left(1-\frac{\alpha}{k+s+1}\right)>0 .
$$

Taking partial derivative of $p$ on both sides of Eq. (30) yields

$$
\frac{\partial\left(\operatorname{var} G_{t+1}\right)}{\partial p}=\frac{(t+s+2-2 \alpha)(t+s)}{(t+s+1)^{2}} \frac{\partial\left(\operatorname{var} G_{t}\right)}{\partial p}+\frac{1-2 E P_{t}}{(t+s+1)^{2}} \frac{\partial\left(E P_{t}\right)}{\partial p} .
$$

The result now follows from the facts that (i) $E P_{t}>1 / 2$ for all $t>0$ if $p \geq 1 / 2$ and $p_{0}>1 / 2$, and (ii) $E P_{t}<1 / 2$ for all $t>0$ if $p \leq 1 / 2$ and $p_{0}<1 / 2$.

Proposition 8. Suppose $F_{t}^{\prime}$ is a bigger process than $F_{t}$ in that $m_{0}^{\prime}=k m_{0}$ and $n_{0}^{\prime}=k n_{0}$, where $k$ is an integer larger than one. In all other respects the two processes are identical.

(i) $E F_{t}^{\prime}<E F_{t}$ for all $t>0$ iff $f_{0}<p$.

(ii) $E F_{t}^{\prime}>E F_{t}$ for all $t>0$ iff $f_{0}>p$.

Proof. When $f_{0}<p$ we have $g_{0}<0$. In this case Eq. (19) shows that $E G_{t}$ is decreasing in $s$. Hence $E F_{t}^{\prime}<E F_{t}$. When $f_{0}>p$ we have $E F_{t}^{\prime}>E F_{t}$ similarly.

Proposition 9. All else equal, the distribution of $F_{t}$ is stochastically increasing, in a strong sense, in $f_{0}$, for all $t>0$.

Proof. We only need to show that $M_{t}$ is stochastically increasing in $f_{0}$ in a strong sense. We prove this by induction. The proposition obviously holds for $t=1$. Suppose $t$ is correct, we prove for $t+1$. Let $M_{t}$ and $M_{t}^{\prime}$ be the two stochastic processes generated by $f_{0}$ and $f_{0}^{\prime}$, assuming that $f_{0}<f_{0}^{\prime}$. By induction hypothesis, there exist $s \leq a<b<c<d \leq t+s$ such that $P\left[M_{t}^{\prime}=\right.$ $i]<P\left[M_{t}=i\right]$ for all integers $i \in[a, b], P\left[M_{t}^{\prime}=i\right]=P\left[M_{t}=i\right]$ for all integers $i \in[b+1, c-1]$ (possibly none), and $P\left[M_{t}^{\prime}=i\right]>P\left[M_{t}=i\right]$ for all integers $i \in[c, d]$. Furthermore, $[a, d]$ covers the support of $M_{t}$ and $M_{t}^{\prime}$. 
For all $i=c+1, \ldots, d+1$, we have

$$
\begin{aligned}
P\left[M_{t+1}^{\prime}=i\right]= & P\left[M_{t}^{\prime}=i-1\right]\left[\alpha p+(1-\alpha) \frac{i-1}{t+s}\right] \\
& +P\left[M_{t}^{\prime}=i\right]\left[\alpha(1-p)+(1-\alpha)\left(1-\frac{i}{t+s}\right)\right] \\
> & P\left[M_{t}=i-1\right]\left[\alpha p+(1-\alpha) \frac{i-1}{t+s}\right] \\
& +P\left[M_{t}=i\right]\left[\alpha(1-p)+(1-\alpha)\left(1-\frac{i}{t+s}\right)\right] \\
= & P\left[M_{t+1}=i\right] .
\end{aligned}
$$

Similarly, for all $i=a, \ldots, b$, we have $P\left[M_{t+1}^{\prime}=i\right]<P\left[M_{t+1}=i\right]$.

To study the relationship between the probability atoms of $M_{t+1}^{\prime}$ and $M_{t+1}$ at the "boundaries", distinguish the following two cases:

Case 1. There are no intermediate "equal" states $(b+1=c)$.

Whatever the relationship between $P\left[M_{t+1}^{\prime}=c\right]$ and $P\left[M_{t+1}=c\right]$, we have $M_{t+1}^{\prime}$ strongly dominates $M_{t+1}$.

Case 2. There exist some intermediate "equal" states $(b+1<c)$.

It can be checked, in a fashion similar to Eq. (37), that (a) $P\left[M_{t+1}^{\prime}=\right.$ $b+1] \leq P\left[M_{t+1}=b+1\right]$, (b) $P\left[M_{t+1}^{\prime}=c\right] \geq P\left[M_{t+1}=c\right]$, and (c) $P\left[M_{t+1}^{\prime}=\right.$ $i]=P\left[M_{t+1}=i\right]$ for all $i=b+2, \ldots, c-1$ (if any). Hence $M_{t+1}^{\prime}$ strongly dominates $M_{t+1}$.

Proposition 10. $\partial\left(\operatorname{var} F_{t}\right) / \partial f_{0}<0$ for all $t>0$ if $p_{0}>1 / 2$ and $p>1 / 2$.

Proof. Using Eq. (31) and Eq. (19), it can be calculated that

$$
\frac{\partial\left(E P_{t}\left(1-E P_{t}\right)\right)}{\partial f_{0}}=\left(1-2 E P_{t}\right)(1-\alpha) \prod_{k=0}^{t-1}\left(1-\frac{\alpha}{k+s+1}\right) \text {. }
$$

If $p_{0}>1 / 2$ and $p>1 / 2$, then because $E P_{t}$ converges to $p$ monotonously, we have $E P_{t}>1 / 2$ for all $t>0$, and therefore

$$
\frac{\partial\left(E P_{t}\left(1-E P_{t}\right)\right)}{\partial f_{0}}<0
$$

for all $t>0$. The result now follows from Eq. (30).

\section{Proposition 11.}

(i) If $1 / 2<\alpha \leq 1$, then as $t \rightarrow \infty, \sqrt{t}\left(F_{t}-p\right)$ converges in distribution to a normal distribution with mean zero and variance $p(1-p) /(2 \alpha-1)$. In particular, $\operatorname{var} F_{t}=O(1 / t)$.

(ii) If $0<\alpha \leq 1 / 2$, then $\operatorname{var} F_{t} \rightarrow 0$ slower than $O(1 / t)$. 
Proof. To understand this proposition, define $v_{t}=(t+s) \operatorname{var} G_{t}$ and rewrite Eq. (30) as

$$
v_{t+1}-v_{t}=\frac{E P_{t}\left(1-E P_{t}\right)-(2 \alpha-1) v_{t}}{t+s+1} .
$$

When $t$ is large, we have

$$
\Delta v_{t+1} \sim \frac{p(1-p)-(2 \alpha-1) v_{t}}{t},
$$

Hence if $2 \alpha-1>0, v_{t}$ is dragged to the limit $p(1-p) /(2 \alpha-1)$.

It is also clear from Eq. (40) that when $0<\alpha<1 / 2$ we have $v_{t} \rightarrow \infty$ in general, so $\operatorname{var} G_{t}$ converges slower than $O(1 / t)$.

A rigorous proof for (i), however, is too technical to be presented here. Various authors have given proofs for general stochastic approximation processes. For references see Blu54, Chu54]. 